\title{
Morfea lineal en golpe de sable: a propósito de un caso
}

\author{
Linear morphea in saber coup: about a case
}

\author{
María Vázquez Sánchez (D), M. Victoria Fuentelsaz del Barrio ${ }^{b}$ (D), Raquel Conejero del Mazo ${ }^{b}$ (D), \\ Cristina Genzor Ríos ${ }^{(\mathbb{D}}$, Mónica López Campos ${ }^{(\mathbb{D})}$, Ana Valer Martínez ${ }^{d}$
}

\begin{abstract}
RESUMEN
La morfea lineal en golpe de sable es una entidad dermatológica caracterizada por la inflamación esclerosante y progresiva del tejido cutáneo en la región frontal y/o en el cuero cabelludo. La cefalea y las crisis convulsivas son dos de los síntomas extracutáneos más frecuentes y están causados por el crecimiento subyacente de la lesión. Es importante un diagnóstico temprano para frenar la progresión e intentar evitar las complicaciones secundarias, principalmente neurológicas. El diagnóstico se basa en el cuadro clínico y el estudio histológico, que permite la confirmación definitiva. El tratamiento de elección es la terapia combinada con corticoides orales y metotrexato. Aun con el tratamiento farmacológico adecuado, esta patología puede presentar un curso recidivante y dejar secuelas a largo plazo. Se presenta el caso de una niña en quien se realizó un diagnóstico rápido de esta enfermedad, a pesar de un cuadro clínico inespecífico. Fue tratada con metotrexato oral con buena respuesta, sin efectos secundarios. Palabras clave: morfea, esclerodermia localizada, pediatría, dermatología.
\end{abstract}

\begin{abstract}
Linear morphea in coup de sabre is a dermatological entity characterized by progressive, sclerosing inflammation of the skin tissue in the frontal region and on the scalp. Headache and seizures are two of the most frequent extracutaneous symptoms and they are caused by the growth of the lesion towards underlying structures. An early diagnosis is important to stop cranial progression and try to avoid secondary complications, mainly neurological. The diagnosis is relied on
\end{abstract}

a. Servicio de Pediatría del Hospital Materno-Infantil Miguel Servet. Zaragoza.

b. Servicio de Dermatología del Hospital Royo Villanova. Zaragoza.

c. Unidad de Pediatría del Centro de Salud Actur Norte. Zaragoza.

d. Unidad de Medicina de Familia y Comunitaria del Centro de Salud Biescas. Huesca.

Correspondencia:

Mónica López Campos: monicalopezmd@hotmail.com

Financiamiento: Ninguno.

Conflicto de intereses: Ninguno que declarar.

Recibido: $10-12-2020$

Aceptado: 31-8-2021 compatible clinical signs and a pathological study that allows a definitive confirmation. The treatment of choice is combination therapy with oral corticosteroids and methotrexate. Despite an adequate pharmacological treatment, this pathology can present a recurrent course and cause long-term sequelae. We present the case of a girl who was diagnosed quickly, despite a not very noticeable symptoms. She has been treated with oral methotrexate with a good response, without side effects. Key words: morphea, localized scleroderma, pediatrics, dermatology.

http:/ / dx.doi.org/10.5546/ aap.2022.e75

Cómo citar: Vázquez Sánchez M, Fuentelsaz del Barrio MV, Conejero del Mazo R, Genzor Ríos C, et al. Morfea lineal en golpe de sable: a propósito de un caso. Arch Argent Pediatr 2022;120(2):e75-e79.

\section{INTRODUCCIÓN}

La morfea o esclerodermia juvenil localizada es una patología caracterizada por la inflamación y fibrosis cutánea. El subtipo morfea lineal (ML) es el más frecuente en la edad pediátrica y suele afectar al sistema musculoesquelético de las extremidades. Entre las variantes de morfea lineal, encontramos la morfea lineal en golpe de sable (MCDS, por su sigla en francés). Se trata de una induración lineal localizada en la región frontal paramedial y en el cuero cabelludo. Suele ser unilateral y de extensión progresiva, y puede afectar al músculo y al tejido óseo. ${ }^{1,2}$

La esclerodermia juvenil localizada es la tercera entidad reumatológica más frecuente en la edad infantil (después de la artritis idiopática juvenil y el lupus eritematoso sistémico), con una incidencia aproximada de 0,4-2,7/ 100000 personas. ${ }^{3}$ Se estima que el $67 \%$ de casos de ML se diagnostica antes de los 18 años. En el caso de la MCDS, el $90 \%$ de los pacientes pediátricos se diagnostican entre los 2 y los 14 años. ${ }^{2}$

Se presenta el caso de una niña a la que se diagnosticó la entidad rápidamente, a pesar de una clínica inespecífica.

\section{CASO CLÍNICO}

Niña de 8 años sin antecedentes patológicos de interés que consultó por una lesión pigmentada de dos meses de evolución y crecimiento progresivo en el cuero cabelludo y la zona frontal. 
A la exploración física, se observaba un área lineal deprimida y poco pigmentada, de $9 \times 1 \mathrm{~cm}$, en la región frontal izquierda, que se extendía hasta la zona parietal izquierda del cuero cabelludo. También se apreciaba una leve induración al tacto, con alopecia en el área afectada (Figura 1).

Ante la sospecha de MCDS, se realizó biopsia cutánea y se indicó tratamiento con tacrolimus pomada $0,03 \%$ y mometasona $1 \%$ crema, en días alternos, a la espera de los resultados de la biopsia.

El estudio histológico evidenció fibras gruesas de colágeno en dermis reticular y papilar, asociadas a un escaso infiltrado inflamatorio intersticial de predominio linfoide y engrosamiento vascular. Se observaban cambios inflamatorios en glándulas ecrinas y unidades pilosebáceas con tendencia a la disposición superficial (Figuras 2 y 3 ).

Tras el diagnóstico de MCDS, se derivó a consulta con los servicios de Oftalmología y Neuropediatría. El estudio oftalmológico, la analítica sanguínea, la resonancia magnética cerebral y la capilaroscopia fueron normales.

Tras revisar la bibliografía, y con manifestaciones clínicas inespecíficas, se decidió comenzar tratamiento con metotrexato a dosis de $12,5 \mathrm{mg} /$ semana oral (comprimidos de 2,5 mg repartidos en 3-0-2 comprimidos al día [mañana-tarde-noche], un único día a la semana) y un comprimido de $5 \mathrm{mg}$ de ácido fólico al día siguiente de haber tomado metotrexato. Además, se añadió tratamiento tópico con calcipotriol y aplicación de betametasona en espuma 2 veces por semana en la zona afectada.

El primer control analítico (bioquímica, hemograma y función hepática) se realizó al mes del inicio del tratamiento, con resultado normal. Posteriormente, se realizaron controles analíticos trimestrales, que también fueron normales. Por otra parte, la paciente presentaba cefaleas ocasionales, sin otra manifestación clínica extracutánea. Tras un año de tratamiento se decidió suspender el metotrexato; persistía una leve pigmentación e induración al tacto. En la actualidad, mantiene controles clínicos semestrales.

\section{DISCUSIÓN}

La Sociedad Europea de Reumatología Pediátrica (PRES, por su sigla en inglés) ha propuesto una clasificación más precisa de morfea en la edad infantil. Actualmente, se consideran los siguientes subtipos: morfea lineal de extremidades o el tronco, morfea lineal de

FIGURA 1. Niña con morfea lineal en golpe de sable en región frontal y en cuero cabelludo. Se observa depresión pigmentada en la región frontal izquierda y cuero cabelludo junto a leve alopecia en el trayecto lineal hacia el cuero cabelludo

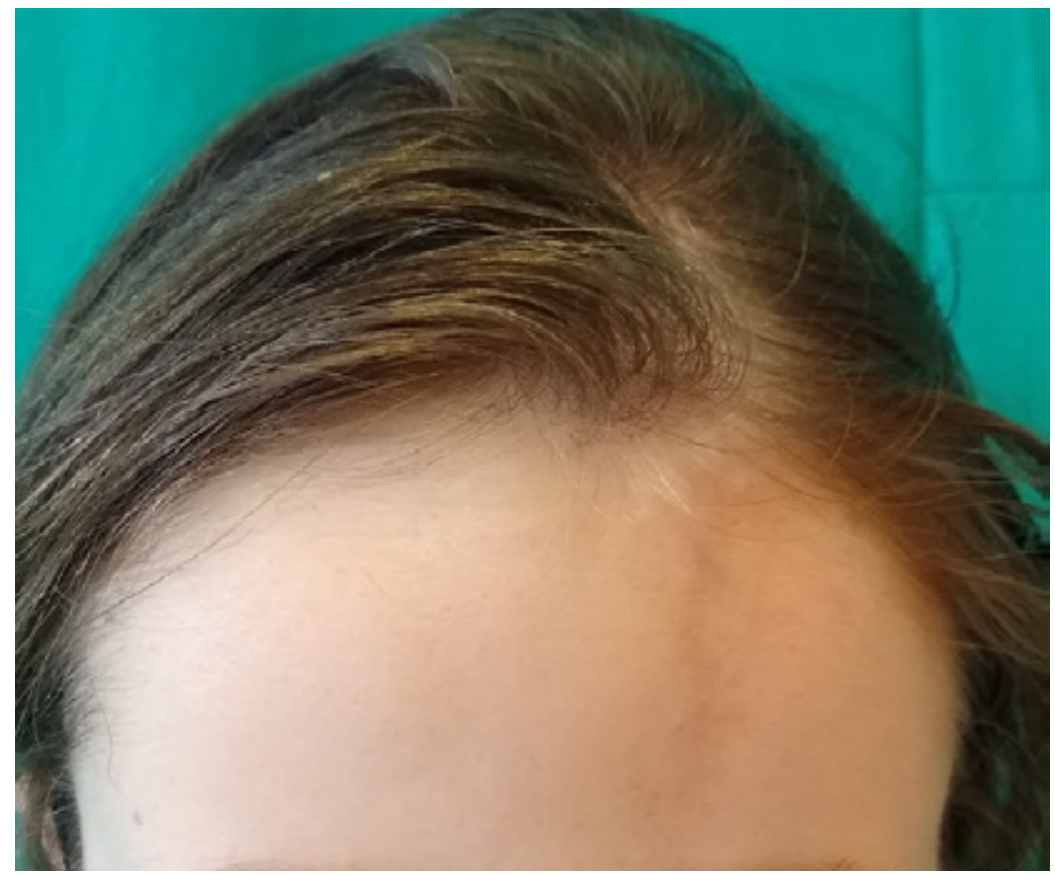


FIGURA 2. Estudio anatomopatológico de la morfea lineal en golpe de sable con tinción hematoxilina-eosina a $2 x$ y 20x. Se observa dermatitis crónica inespecífica asociada a engrosamiento de las fibras de colágeno, compatible con la fase temprana de morfea

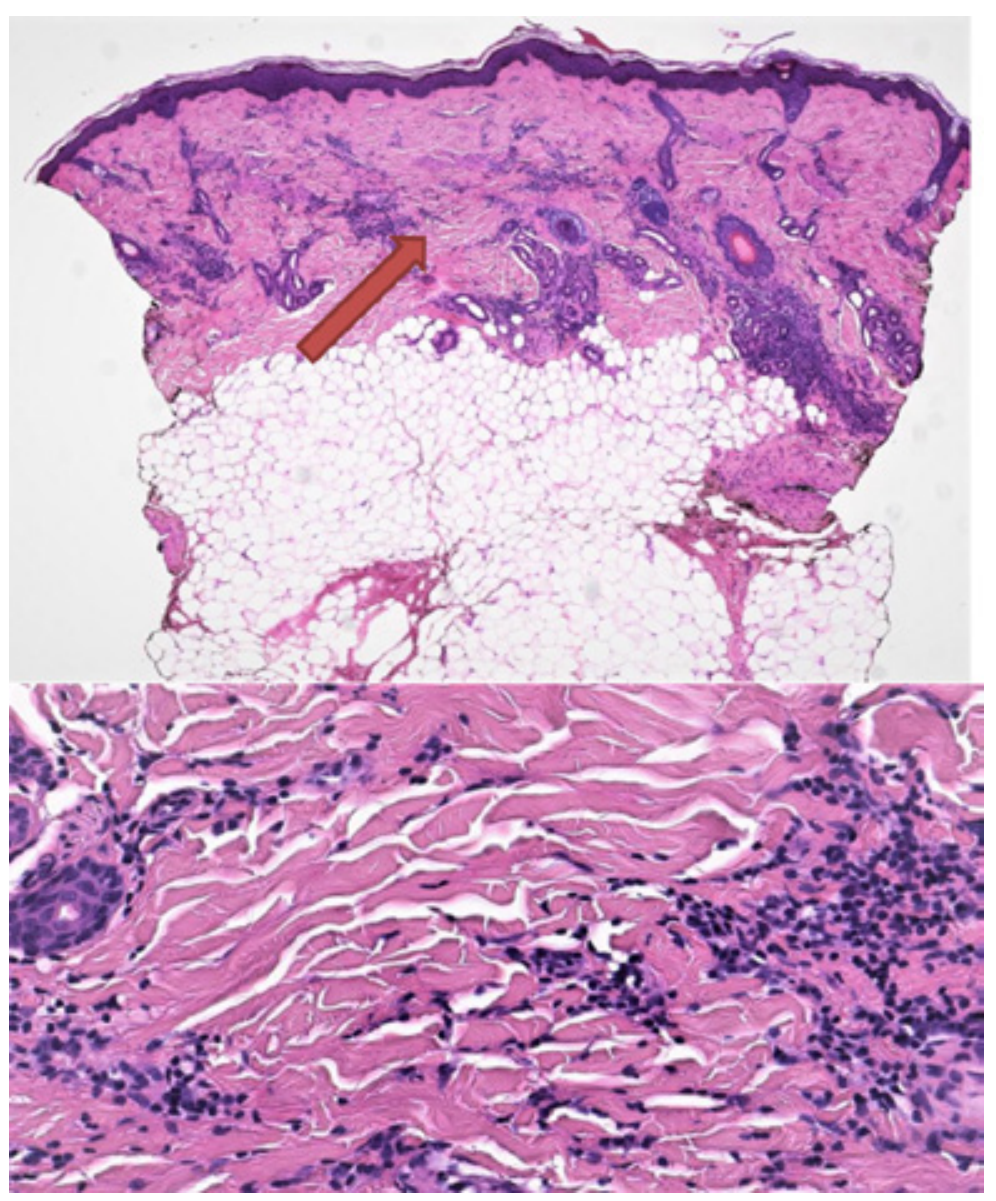

FIgURA 3. Histología de morfea lineal en golpe de sable con tinción tricrómica de Masson a $4 x$ donde se observa la coloración azulada de las fibras de colágeno en un fragmento de piel compatible con morfea

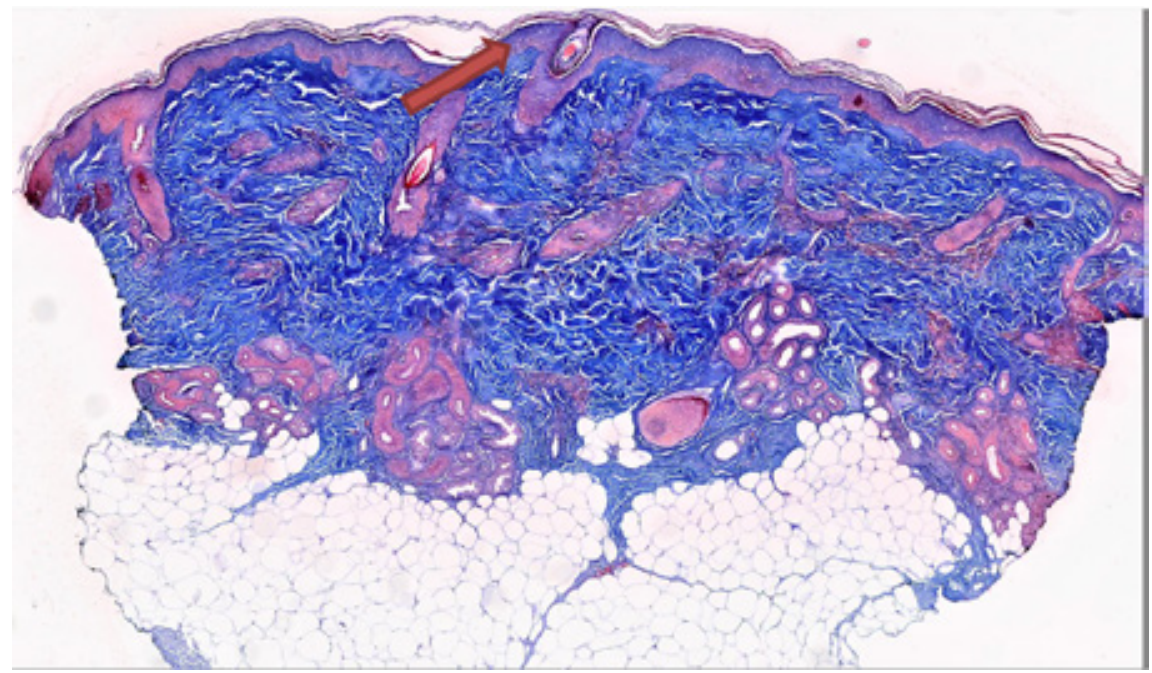


cuero cabelludo y la cara (incluye MCDS y el síndrome de Parry-Romberg o hemiatrofia facial progresiva), morfea circunscrita o en placas, morfea mixta, morfea generalizada y morfea panesclerótica (Tabla 1). La MCDS es un subtipo de morfea lineal localizada en la cabeza y en el área facial. Se trata de una patología dermatológica, más frecuente en las niñas, que suele debutar en edad infantil. Más del $60 \%$ de los afectados se diagnostican antes de los 18 años. ${ }^{2,3}$

Se desconoce su etiología, aunque parecen intervenir factores ambientales y genéticos. En ocasiones, existen antecedentes personales y/o familiares de enfermedades autoinmunitarias y reumatológicas, como esclerodermia, artritis reumatoide, lupus eritematoso sistémico y tiroiditis. $^{2}$

En una minoría de casos, se han descrito factores ambientales concomitantes, como traumatismos mecánicos, picaduras, vacunas, radiaciones, fármacos, infecciones o estrés psicógeno. ${ }^{1-3}$

Suele iniciarse con una induración lineal en la región frontal y en el cuero cabelludo, que progresa hacia alopecia localizada, atrofia y depresión cutánea, con posible afectación del tejido subyacente.

El diagnóstico es clínico y se confirma de forma definitiva mediante el estudio anatomopatológico.
El diagnóstico puede demorarse años y confundirse con pigmentaciones o cicatrices antiguas. Otros diagnósticos diferenciales incluyen ciertas dermatosis lineales adquiridas o congénitas, como nevo epidérmico, liquen plano lineal, laporoqueratosis lineal o alopecia cicatricial. ${ }^{4}$

El síndrome de Parry-Romberg o atrofia hemifacial progresiva (SPR, por su sigla en inglés) es otra forma de ML que afecta al polo cefálico y que consiste en una pérdida de tejido dérmico, tejido celular subcutáneo, musculatura facial y lingual; puede alcanzar la glándula parótida y el hueso en el lado inferior de la cara. Afecta a un lado de la cara, en general debajo de la frente. La superposición de la MCDS y el SPR se puede observar en algunos casos y algunos autores creen que su asociación es más frecuente de lo esperado. ${ }^{2,3}$

La detección temprana es imprescindible, tanto para limitar su progresión como para evitar complicaciones ya que, a menudo, se acompaña de afectación extracutánea.

El síntoma extracutáneo más habitual es la cefalea, seguido de crisis epilépticas y alteraciones oftalmológicas palpebrales y del segmento anterior (uveítis y epiescleritis). La afectación oral es infrecuente, aunque se han descrito casos con áreas blancas fibróticas de aspecto cicatricial en cavidad bucal, lengua depapilada, recesión gingival y resorción ósea alveolar. ${ }^{3}$

Tabla 1. Resumen de las distintas formas de morfea en la edad infantil

\begin{tabular}{|c|c|}
\hline Subtipo & Descripción \\
\hline $\begin{array}{l}\text { Morfea lineal }(\mathrm{ML}) \\
\text { Forma más frecuente en la infancia }(51-65 \%)\end{array}$ & $\begin{array}{l}\text { ML de las extremidades o el tronco } \\
\text { ML del cuero cabelludo y la cara } \\
\text { - Morfea en golpe de sable. } \\
\text { - Síndrome de Parry-Romberg o atrofia hemifacial progresiva. }\end{array}$ \\
\hline Morfea en placas (26-37 \%) & $\begin{array}{l}\text { Placas redondeadas u ovaladas, induradas, con halo violáceo } \\
\text { circundante. } \\
\text { - Superficial (fibrosis limitada a la dermis). } \\
\text { - Profunda (puede extenderse a la fascia muscular y al hueso). }\end{array}$ \\
\hline Morfea mixta (15\%) & Combinación de 2 o más subtipos \\
\hline Morfea generalizada $(7-8 \%)$ & $\begin{array}{l}\text { Presencia de placas de morfea en número } \geq 4 \text {, de diámetro }>3 \mathrm{~cm} \text {, } \\
\text { que van agrupándose hasta alcanzar al menos } 2 \text { de } 7 \text { sitios } \\
\text { anatómicos distintos. Para algunos autores, debe afectar }>30 \% \text { de la } \\
\text { superficie corporal. }\end{array}$ \\
\hline Morfea panesclerótica $(0,27 \%)$ & $\begin{array}{l}\text { Afectación circunferencial de todo el espesor de la piel, subcutáneo, } \\
\text { músculo y hueso. Pueden aparecer úlceras o carcinomas } \\
\text { epidermoides. }\end{array}$ \\
\hline
\end{tabular}


En el estudio radiológico, se pueden evidenciar diferentes hallazgos patológicos no patognomónicos, como adelgazamiento del tejido óseo subyacente, atrofia cerebral focal, deformidades óseas (adelgazamiento en la región suprayacente), desdibujamiento de sustancia gris y blanca, alteraciones de señal de T2 bajo la región afectada, calcificaciones o infartos cerebrales. ${ }^{2,5}$

Recientemente un grupo de radiólogos estadounidenses ha demostrado, mediante imágenes de perfusión por resonancia magnética e imágenes espectroscópicas, una disminución del volumen sanguíneo cerebral, un aumento del tiempo medio de tránsito y una disminución de los metabolitos en una paciente con MLCS. ${ }^{6}$

Las alteraciones analíticas no parecen correlacionarse con la actividad y el pronóstico de la enfermedad. En las morfeas profundas, es más habitual encontrar elevación de reactantes de fase aguda, leucocitosis y aumento de la velocidad de sedimentación. En algunos casos, se han demostrado niveles altos de inmunoglobulinas (IgG) A y M. Se describe también la presencia de elevación del factor reumatoide, trombocitopenia, eosinofilia o hipocomplementemia en algunos pacientes. Los anticuerpos anticentrómero y antiSL70 no suelen ser positivos; mientras que, en los anticuerpos antinucleares (ANA), la positivdad puede alcanzar hasta el $50 \%$. 1,2,5

La MCDS debe considerarse una forma moderada y/o grave de la enfermedad, por lo que es conveniente el tratamiento sistémico en fases iniciales para evitar su progresión, incluso en aquellos casos de escasa expresividad, como el de la paciente aquí presentada. Existen diferentes pautas de tratamiento con metotrexato (solo o asociado a corticoides por vía oral o intravenosa); la vía subcutánea es la preferida por la mayoría de profesionales. ${ }^{2,6}$ Nosotros optamos por la vía oral porque la paciente presentaba temor a las agujas, aunque es cierto que su buena tolerancia oral permitió continuar con el tratamiento por esta vía, en lugar de la vía subcutánea. La duración del tratamiento con metotrexato fue de un año, aunque puede ser variable según la bibliografía. ${ }^{2}$ Tras este tiempo, se observó menor induración, tamaño similar y ausencia de progresión.
El tratamiento con otros fármacos, como el tacrolimus o el mofetil-micofenolato, cuenta con escasa evidencia científica en pediatría. ${ }^{2}$ Se han documentado casos de tratamiento con inyecciones intralesionales con acetónido de triamcinolona en dosis de $5 \mathrm{mg} / \mathrm{ml}$ una vez al mes durante 3 meses con mejoría o detención de la progresión. También se ha empleado la hidroxicloroquina, colchicina, vitamina $\mathrm{E}$ a dosis elevadas, D-penicilamina, interferón gamma por vía subcutánea asociada a carbamacepina, tacrolimus tópico, análogos de la vitamina D (calcitriol y el calcipotriol) y corrección quirúrgica, con resultados variables. ${ }^{7}$

Aunque se han validado varias escalas clínicas como el puntaje de piel modificado (MSS, por su sigla en inglés) y el puntaje de pie modificado de Rodnan (MRSS, por su sigla en inglés), pocos son los reumatólogos y dermatólogos que las emplean en su práctica diaria. ${ }^{2}$

La mayoría de los autores concuerdan en que la MCDS es una patología de curso impredecible e incluso crónico, con altas tasas de secuelas neurológicas, oftalmológicas y estéticas. Por tanto, es importante el seguimiento estrecho, la derivación a otros especialistas y el tratamiento en fases iniciales con el objetivo de frenar el avance de la enfermedad.

\section{REFERENCIAS}

1. Zulian F, Athreya BH, Laxer R, Nelson AM, et al. Juvenile localized scleroderma: clinicaland epidemiological features in 750 children. An internationalstudy. Rheumatology (Oxford). 2006; 45(5):614-20.

2. Aranegui B, Jiménez-Reyes J. Morfea en la infancia: actualización. Actas Dermosifiliogr (Engl Ed). 2018; 109(4):312-22.

3. Vieira Martins M, Azevedo I, Rodrigues C, Oliveira M. Linear scleroderma en coup de sabre; a diferente clinical presentation. Acta Reumatol Port. 2021; 46(1):72-6.

4. Weibel L, Laguda B, Atherton D, Harper JI. Misdiagnosis and delay in referral of children with localized scleroderma. Br JDermatol. 2011; 165(6):1308-13.

5. Holland KE, Steffes B, Nocton JJ, Schwabe MJ, et al. Linear scleroderma en coup de sabre with associated neurologic abnormalities. Pediatrics. 2006; 117(1):e132-6.

6. Nguyen K, Atty C, Ree A. Linear scleroderma in coup de sabre presenting with seizures. Radiol Case Rep. 2020; 15(11):2164-70.

7. Rattanakaemakorn P, Jorizzo JL. The efficacy of methotrexate in the treatment of en coup de sabre (linear morphea subtype). J Dermatolog Treat. 2018; 29(2):197-9. 